\title{
SÍNTESIS DE LOS APHYLLOPHORALES S.L. LIGNÍCOLAS (BASIDIOMYCOTA, MACROFUNGI) PRESENTES EN LAS COMUNIDADES VEGETALES DE ANDALUCÍA
}

\author{
Antonio ORTEGA y Juan LORITE
}

\begin{abstract}
RESUMEN. Síntesis de los Aphyllophorales s.l. lignícolas (Basidiomycota, Macrofungi) presentes en las comunidades vegetales de Andalucía. Se analiza el comportamiento ecológico (sustrato) del componente micológico (Aphyllophorales lignícolas) que fructifica en las comunidades vegetales naturales de Andalucía. Se analiza la distribución cuantitativa por termotipos, ombrotipos, sustratos y formaciones vegetales. Se establece el grado de afinidad por el sustrato de las especies y géneros mejor representados.
\end{abstract}

Palabras clave. Ecología de hongos, Aphyllophorales, forófitos, España.

ABSTRACT. Synthesis of lignicolous Aphyllophorales (Basidiomycota, Macrofungi) on natural plant communities from Andalusia (Spain). The ecological behaviour of the mycological component (lignicolous A phyllophorales) that fruits in natural plant communities in Andalusia is analysed. Furthermore, quantitative distribution by thermotypes, ombrotypes, substract and physiognomic plant formations is analysed. The substract affinity level of species and genera best represented is established.

Key words. Ecological behaviour, lignicolous Aphyllophorales; thermotype, ombrotype, substract, plant communities, affinity.

\section{INTRODUCCIÓN}

Hasta la fecha se han realizado numerosos estudios sobre los Aphyllophorales lignícolas de la Península Ibérica (en menor medida en Andalucía), todos ellos centrados exclusivamente en aspectos taxonómicos y florísticos, estos últimos referentes a la localidad de recolección (UTM, altitud, etc..) y sustrato sobre el que se desarrolla cada uno de los taxones. Sin embargo, no existe hasta la fecha ningún trabajo de recopilación a nivel de Andalucía de todos estos datos (publicados e inéditos), en los que se relacione la micoflora de Aphyllophorales con los tipos de vegetación y se estudie su distribución cuantitativa por sustratos y tipos de vegetación, así como la afinidad de los distintos taxones por los distintos sustratos. Es posible que determinadas formaciones vegetales estén poco estudiadas, y por tanto los datos que se aportan puedan ser matizados o completados en el futuro; no obstante, este trabajo puede suponer una primera aproximación, en la que se aportan datos interesantes de cara al comportamiento de este grupo fúngico. 


\section{MATERIAL Y MÉTODOS}

Como punto de partida se ha utilizado el material depositado en distintos herbarios $(\mathrm{AH}$, GDAC, MHG, JA-F, MA-Fungi y AMSS), así como numerosas referencias bibliográficas (Calonge \& Oria de Rueda, 1988; Calonge \& Romero de la Osa, 1997; Calonge \& Tellería, 1980; Gómez et al., 1995; Hjortstam et al., 1981, 1988; Honrubia \& Llimona, 1982; Jiménez-Antonio, 1994; Malençon, 1968; Malençon \& Bertault, 1976; Malençon \& Llimona, 1980; Manjón \& Moreno, 1983; Moreno \& Manjón, 1987; Ortega et al., 1982, 1991, 1996, 1997; Romero-de la Osa, 1992, 1993, 1996; Tellería, 1984, 1985, 1987, 1990, 1991; Vizoso \& Ortega, 1992; Vizoso et al., 1991). En total se han analizado 1320 muestras correspondientes a 271 especies (anexo 1), que constituyen la diversidad de Aphyllophorales lignícolas de Andalucía (según los datos registrados hasta el momento).

A partir de los datos anteriores se han estudiado los siguientes aspectos:

a) Termotipo y ombrotipo: Con los datos de recolección (Localidad, altitud, UTM), se ha asignado un termotipo y un ombrotipo (RivasMartínez, 1987; 1996) para cada una de las muestras, de manera que sea posible determinar la riqueza tanto en especies como en muestras de cada uno de los termotipos y ombrotipos presentes en Andalucía.

b) Distribución cuantitativa por sustratos tanto de especies como de muestras; el primer dato nos aporta la riqueza de especies en el sustrato, mientras que el segundo nos proporciona una idea relativa de la abundancia de Aphyllophorales en el sustrato y por tanto de la "idoneidad" del sustrato para el desarrollo de este grupo.

c) Distribución cuantitativa por tipos fisiognómicos de vegetación.

d) Para estudiar el nivel de afinidad de cada especie por un determinado sustrato, hemos procedido de la siguiente forma: En primer lugar el número de sustratos se ha reducido a 25, que son los que presentan un número significativo de muestras recolectadas. En cuanto a las especies se han eliminado las representadas por menos de 5 muestras, con 10 que se ha reducido el número total de especies a 75 (señaladas con * en el anexo 1). Posteriormente se ha calculado el porcentaje de presencia de cada especie en los distintos sustratos: Índice de presencia $=\left(\mathrm{n}^{\circ}\right.$ de veces que la especie aparece en el sustrato $/ n^{\circ}$ total de veces que aparece la especie) x 100 (adaptado de Goslee et al., 1997). Con el resultado de este cálculo se ha asignado una categoría de afinidad, con el siguiente criterio: Estrictamente ligado (El) $>99 \%$, Fuertemente ligado (Fl) 67-99 \%, Laxamente ligado (Ll) 34-66 \%, Ocasional (Oc) 5-33\%, Accidental (Ac) $<5 \%$.

e) Para determinar la afinidad existente a nivel genérico se han eliminado los géneros poco representados (siguiendo el mismo criterio que para las especies y por supuesto eliminando los géneros constituidos por una sola especie, puesto que el resultado sería el mismo que a nivel de especie), quedando un total de 31 géneros, a los que se ha asignado una categoría de afinidad por los distintos sustratos.

\section{RESULTADOS Y DISCUSIÓN}

Se han registrado un total de 271 especies cuya distribución a lo largo de los parámetros considerados se discute a continuación.

Observando la distribución por termotipos y ombrotipos (tab.1), cabe destacar que el mesomediterráneo presenta 244 de las 271 especies registradas para Andalucía, así como 1087 muestras de las 1320 totales, ante este hecho cabe una doble explicación, por una parte se trata del termotipo más extendido en Andalucía y por otra, parece presentar las mejores condiciones para el desarrollo de este grupo. En el extremo contrario podemos 


\begin{tabular}{lcc}
\hline & $\mathbf{n}^{\mathbf{0}}$ de especies & $\mathbf{n}^{\mathbf{0}}$ de muestras \\
\hline Termotipos & & \\
Termomediterráneo & 82 & 167 \\
Mesomediterráneo & 244 & 1087 \\
Supramediterráneo & 53 & 65 \\
Oromediterráneo & 1 & 1 \\
Ombrotipos & & \\
Semiárido & 46 & 88 \\
Seco & 84 & 139 \\
Subhúmedo & 213 & 752 \\
Húmedo & 99 & 195 \\
Hiperhúmedo & 63 & 146 \\
\hline
\end{tabular}

Tabla 1. Distribución de especies y muestras en los distintos termotipos y ombrotipos presentes en Andalucía. Distribution of species and records in each thermotype and ombrotype present in Andalusia.

\begin{tabular}{|c|c|c|}
\hline Sustratos & Especies & Muestras \\
\hline Quercus ilex subsp. ballota & 122 & 360 \\
\hline Quercus suber & 65 & 143 \\
\hline Abies pinsapo & 47 & 133 \\
\hline Quercus faginea & 52 & 71 \\
\hline Populus spp. & 30 & 71 \\
\hline madera sin identificar & 35 & 45 \\
\hline Pinus spp. & 21 & 43 \\
\hline Tamarix spp. & 20 & 42 \\
\hline Cistus spp. & 19 & 37 \\
\hline Planifolios & 32 & 35 \\
\hline Pinus halepensis & 21 & 33 \\
\hline Quercus spp. & 14 & 20 \\
\hline Castanea sativa & 15 & 18 \\
\hline Olea europaea & 13 & 18 \\
\hline Pinus nigra subsp. salzmannii & 10 & 13 \\
\hline Alnus glutinosa & 9 & 13 \\
\hline Pinus pinaster & 9 & 11 \\
\hline Quercus canariensis & 7 & 11 \\
\hline Ulmus minor & 10 & 10 \\
\hline Chamaerops humilis & 9 & 10 \\
\hline madera quemada & 9 & 10 \\
\hline Ulex parviflorus & 8 & 10 \\
\hline Ulex baeticus & 7 & 9 \\
\hline Phoenix spp. & 5 & 9 \\
\hline Fraxinus angustifolia & 8 & 8 \\
\hline Ceratonia siliqua & 8 & 8 \\
\hline Arbutus unedo & 8 & 8 \\
\hline Juniperus phoenicea & 7 & 8 \\
\hline Eucalyptus spp. & 6 & 8 \\
\hline Rosmarinus officinalis & 7 & 7 \\
\hline
\end{tabular}

Tabla 2. Distribución por sustratos (30 sustratos con mayor riqueza) de especies y muestras, considerando el total de especies registradas en Andalucía (271 especies; ordenadas de más a menos abundante). Distribution in substracts (30 substracts with more richness) of species and records, considered the total of recorded species in Andalusia (271 species; in decrease order). destacar el oromediterráneo en el que tan solo se ha registrado una especie, aún teniendo en cuenta que la representación espacial de este termotipo es menor que la de los demás, la baja diversidad registrada hace pensar en limitaciones de carácter climático para la fructificación de Aphyllophorales.

En cuanto a los termotipos, aunque el seco es el más ampliamente extendido en Andalucía, es el subhúmedo el que presenta una mayor riqueza (213 especies, 752 muestras), es de destacar también que el húmedo y el hiperhúmedo, a pesar de tener una extensión muy reducida en Andalucía (sobre todo en el caso del hiperhúmedo), presentan mayor diversidad que el semiárido y una diversidad comparable a la registrada en el seco.

En la tabla 2 vemos la distribución por sustratos, en la que podemos destacar que casi la mitad de especies aparecen en Quercus ilex subsp. ballota, así como, que la mayor representación tanto en especies como en muestras, aparecen en el género Quercus, con la excepción de Abies pinsapo que presenta 47 especies, siendo la extensión de esta formación en Andalucía de 2.350 ha en total (Arista et al., 1997).

\begin{tabular}{lcc}
\hline Tipos fisiognómicos & especies & muestras \\
\hline Bosques mediterráneos & 140 & 518 \\
Bosques marcescentes & 92 & 225 \\
Matorrales arbustivos mediterráneos & 64 & 127 \\
Bosques riparios & 42 & 105 \\
Matorrales silicícolas & 27 & 44 \\
Cultivos y ornamentales & 21 & 35 \\
Vegetación riparia cauces intermitentes 20 & 42 \\
Matorrales calcícolas & 19 & 30 \\
Orlas de espinosas de bosque & 11 & 14 \\
Pinares-sabinares de montaña & 11 & 15 \\
No diferenciados & 60 & 107 \\
Otros & 47 & 58
\end{tabular}

Tabla 3. Distribución de especies y muestras por tipos fisionómicos (sobre las 271 especies registradas; ordenadas de más a menos abundante). Distribution of species and records by physiognomic types (over 271 recorded species; in decrease order). 


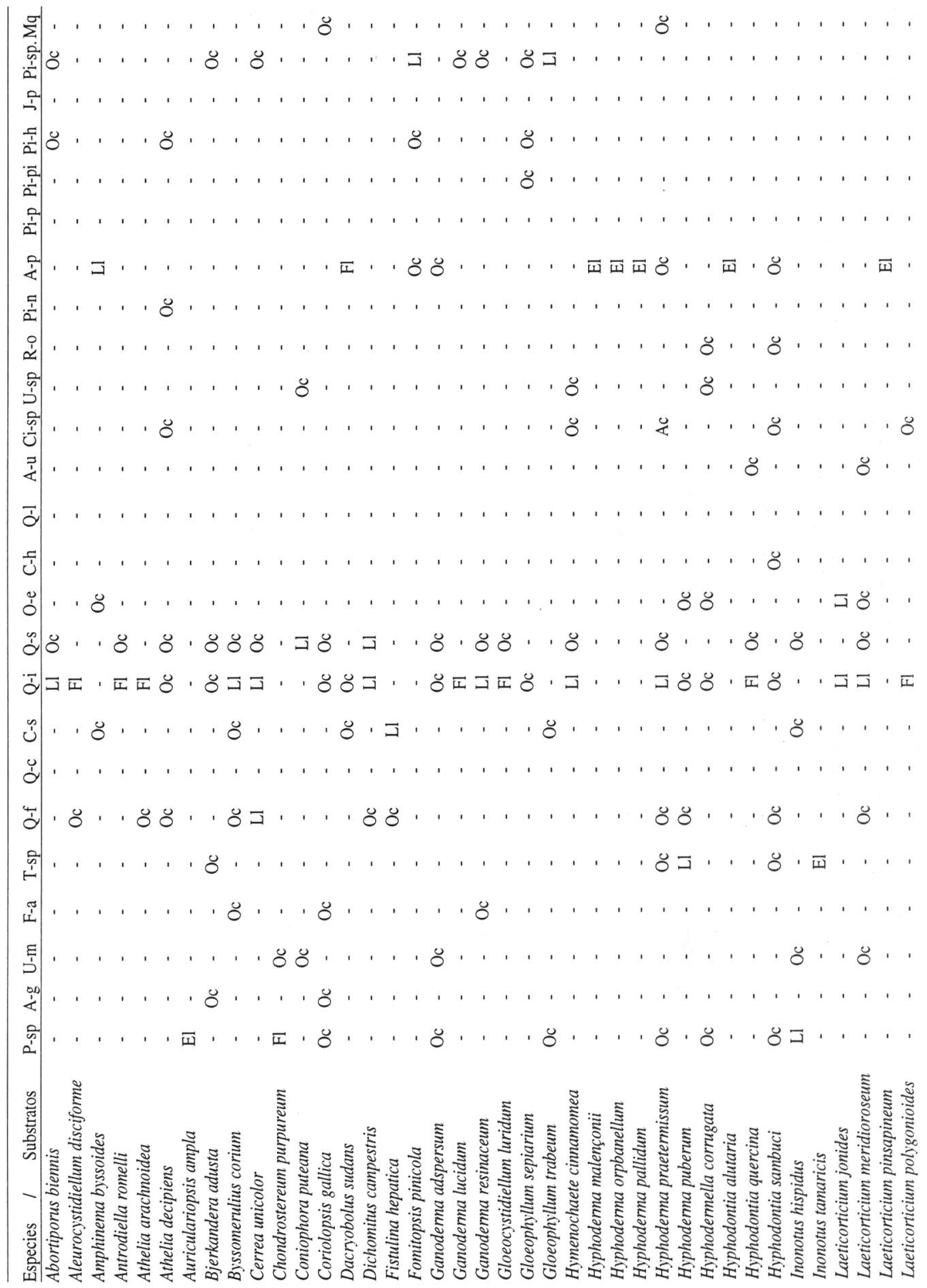




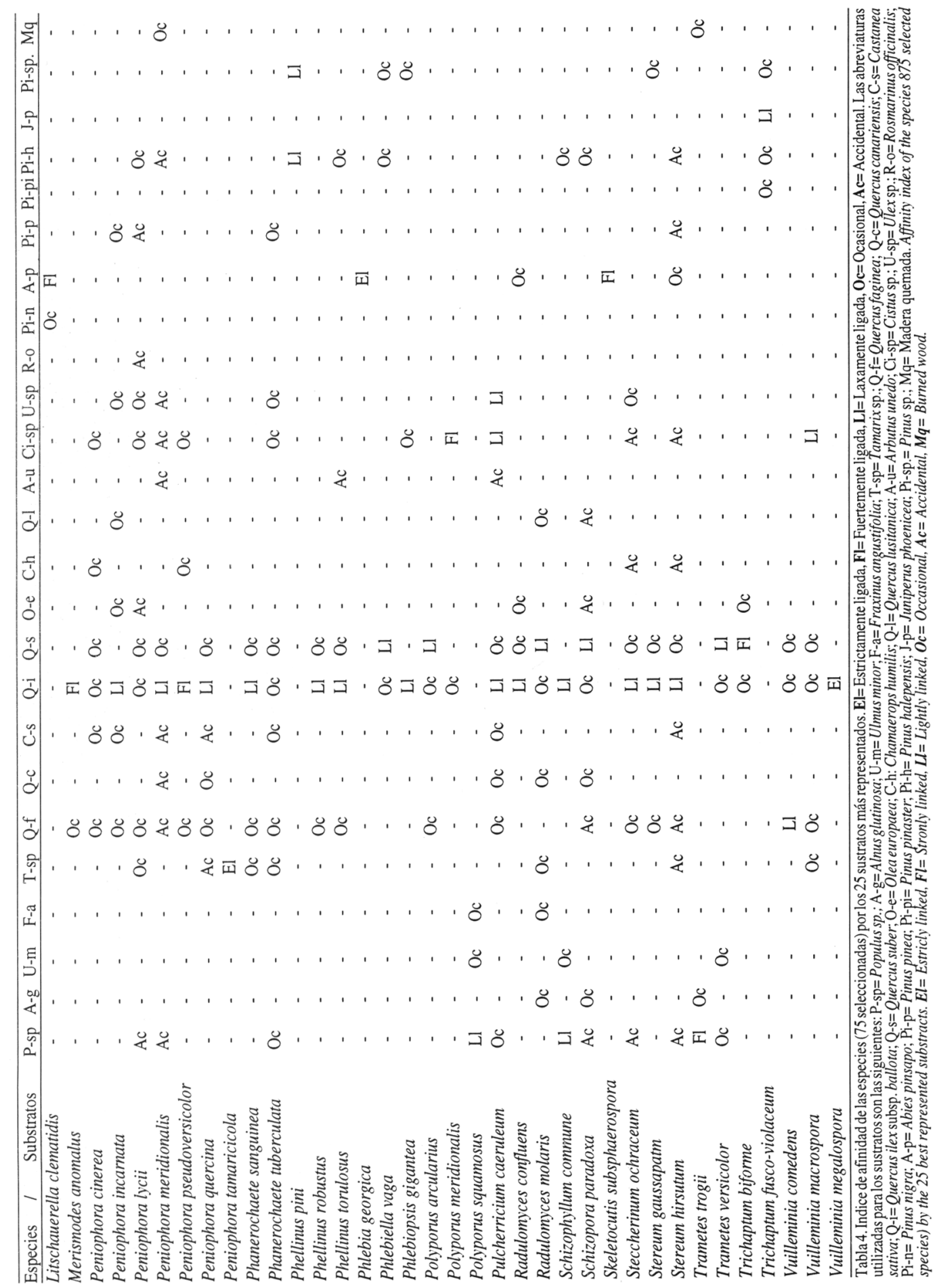




\begin{tabular}{lccccc}
\hline & El & Fl & Ll & Oc & Ac \\
\hline Populus sp. & 1 & 2 & 3 & 9 & - \\
Alnus glutinosa & - & - & - & 5 & - \\
Ulmus minor & - & - & - & 8 & - \\
Fraxinus angustifolia & - & - & - & 5 & - \\
Tamarix sp. & 2 & - & 1 & 8 & 2 \\
Quercus faginea & - & - & 2 & 25 & 3 \\
Quercus canariensis & - & - & - & 4 & 1 \\
Castanea sativa & - & - & 1 & 9 & 3 \\
Quercus ilex subsp. ballota & 1 & 9 & 22 & 21 \\
Quercus suber & - & 1 & 7 & 20 & - \\
Olea europaea & - & - & 1 & 7 & 2 \\
Chamaerops humilis & - & - & - & 3 & 2 \\
Quercus lusitanica & - & - & - & 2 & 1 \\
Arbutus unedo & - & - & - & 2 & 3 \\
Cistus sp. & - & 1 & 2 & 9 & 4 \\
Ulex sp. & - & - & 1 & 7 & 1 \\
Rosmarinus officinalis & - & - & - & 2 & 1 \\
Pinus nigra & - & - & - & 2 & - \\
Abies pinsapo & 6 & 3 & 1 & 6 & - \\
Pinus pinea & - & - & - & 2 & 2 \\
Pinus pinaster & - & - & 1 & 7 & 2 \\
Pinus halepensis & - & - & 1 & 4 & - \\
Juniperus phoenicea & - & - & 1 & - & - \\
Pinus sp. & - & - & 3 & 10 & - \\
Madera quemada & - & - & - & 4 & - \\
Pat & & & & &
\end{tabular}

Tabla 5. Sustratos por categorías de afinidad para especies (75 especies seleccionadas). $\mathbf{E l}=$ Estrictamente ligada, $\mathbf{F l}=$ Fuertemente ligada, $\mathbf{L l}=$ Laxamente ligada, $\mathbf{O c}=$ Ocasional, Ac = Accidental. Substracts by affinity index for species (75 selected species). $\boldsymbol{E} \mathbf{l}=$ Estricly linked, $\boldsymbol{F} \mathbf{l}=$ Stronly linked, $\boldsymbol{L} \boldsymbol{l}=$ Lightly linked, $\boldsymbol{O} \boldsymbol{c}=$ Occasional, $\boldsymbol{A} \boldsymbol{c}=$ Accidental.

En cuanto a la distribución por formaciones vegetales de especies y muestras (tab. 3), simplemente destacar la importancia de los bosques esclerófilos. Este resultado es lógico, por su abundancia dentro del área de estudio, sin embargo, aunque los matorrales calcícolas y silicícolas, son más abundantes que las formaciones de bosques esclerófilos, no presentan una gran diversidad en especies.

En la tabla 4 vemos el resultado de aplicar los índices de afinidad a las 75 especies representadas con más de cinco muestras. Para hacernos una idea más clara de cuantas especies de cada una de las categorías (Estrictamente ligada, fuertemente ligada, laxamente ligada, ocasional y accidental) aparecen en los distintos sustratos, hemos elaborado a partir de la tabla anterior la tabla 5 , en la que podemos ver que existen solo 10 especies estrictamente ligadas a algún sustrato de donde es posible destacar el sustrato Abies pinsapo que posee 6 de las 10 especies estrictamente ligadas, lo que nos indica una elevada especificidad en comparación con el resto de sustratos. Si nos fijamos en las fuertemente ligadas podemos destacar Quercus ilex subsp. ballota, que posee $\overline{9}$ especies fuertemente ligadas, es decir que 9 de las 75 especies, están presentes mayoritariamente en este sustrato. Si nos fijamos en los totales la mayoría de las especies aparecen de forma ocasional en un determinado sustrato, es decir que se desarrollan sobre distintos sustatos de forma más o menos ubiquista.

\begin{tabular}{lccccc}
\hline & El & Fl & Ll & Oc & Ac \\
\hline Populus sp. & - & - & 1 & 6 & 8 \\
Alnus glutinosa & - & - & 1 & 2 & 2 \\
Ulmus minor & - & - & - & 3 & 3 \\
Fraxinus angustifolia & - & - & - & - & 4 \\
Tamarix sp. & - & - & 1 & 6 & 4 \\
Quercus faginea & - & - & - & 12 & 3 \\
Quercus canariensis & - & - & - & 1 & 2 \\
Castanea sativa & - & - & - & 3 & 4 \\
Quercus ilex subsp. ballota & - & 2 & 11 & 13 & 1 \\
Quercus suber & - & - & 2 & 22 & 1 \\
Olea europaea & - & - & - & 2 & 5 \\
Chamaerops humilis & - & - & - & - & 4 \\
Quercus lusitanica & - & - & - & - & 2 \\
Arbutus unedo & - & - & - & - & 7 \\
Cistus sp. & - & - & 1 & 7 & 7 \\
Ulex sp. & - & - & - & 3 & 3 \\
Rosmarinus officinalis & - & - & - & 1 & 2 \\
Pinus nigra & - & - & 1 & 4 & 2 \\
Abies pinsapo & - & 2 & 3 & 6 & 3 \\
Pinus pinea & - & - & - & 2 & 3 \\
Pinus pinaster & - & - & - & 2 & - \\
Pinus halepensis & - & - & - & 6 & 4 \\
Juniperus phoenicea & - & - & - & 1 & 2 \\
Pinus sp. & - & - & 1 & 6 & 2 \\
Madera quemada & - & - & - & 1 & 4 \\
Made & & & & &
\end{tabular}

Tabla 7. Sustratos por categorías de afinidad para géneros (75 especies seleccionadas). $\mathbf{E l}=$ Estrictamente ligada, $\mathbf{F l}=$ Fuertemente ligada, $\mathbf{L l}=$ Laxamente ligada, $\mathbf{O c}=$ Ocasional, $\mathbf{A c}=$ Accidental . Substracts by affinity index for genera ( 75 selected species). = Estricly linked, $\boldsymbol{F l}=$ Stronly linked, $\boldsymbol{L} \boldsymbol{l}=$ Lightly linked, $\boldsymbol{O c}=$ Occasional, $\boldsymbol{A c}=$ Accidental (see correspondence in the foot of table 4). 


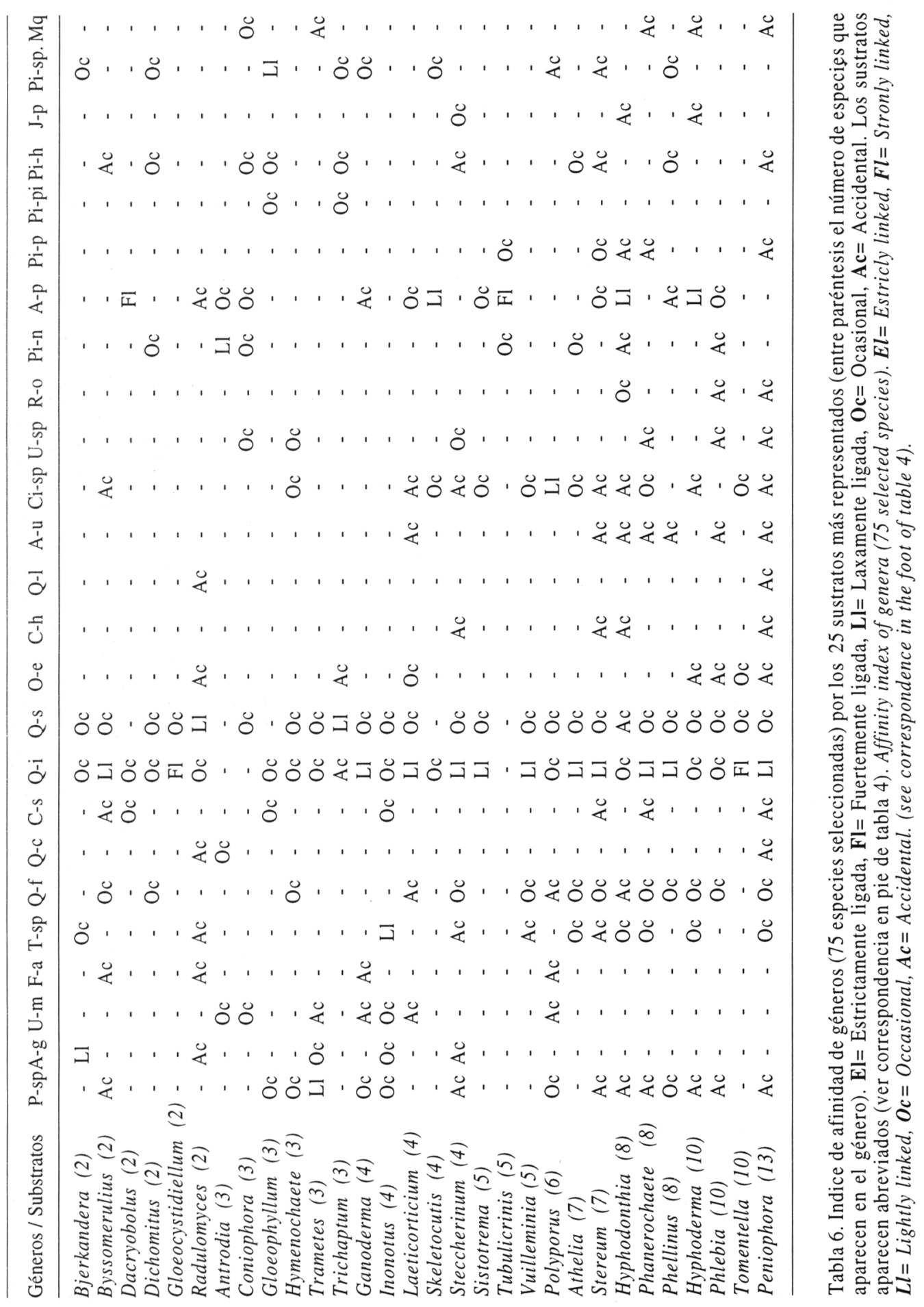


En cuanto al índice de afinidad por géneros sustratos (tab.6), hemos elaborado igual que en el apartado anterior una tabla de categorías de afinidad por sustratos (tab.7), donde podemos observar que no hay ningún género estrictamente ligado a ningún sustrato, aunque si existen 4 géneros fuertemente ligados, 2 a Quercus ilex subsp. ballota (Gloeocystidiellum y Tomentella) y dos a Abies pinsapo (Dacryobolus y Tubulicrinis), mientras que a este nivel (de género) en la mayoría de los casos la presencia en un determinado sustrato es ocasional.

\section{ANEXO}

Listado de los Aphyllophorales, recogidos en el presente estudio. Las especies marcadas con un * figuran entre las 75 estudiadas en las tablas 4, 5, 6 y 7. Se indican las provincias donde han sido recolectadas: AL: Almería; CA: Cádiz; CO: Córdoba; GR: Granada; HU: Huelva; J: Jaén; MA: Málaga y SE: Sevilla.

\section{APHYLLOPHORALES NO PORADOS:}

Acanthophysium minor (Pilát) Tellería; GR.

*Aleurocystidiellum disciforme (DC.: Fr.) Tellería; CA, CO, GR, HU. J.

Aleurodiscus cerussatus (Bres.) Höhn. \& Litsch.; MA. Aleurodiscus dextrinoideocerussatus Manjón, Blanco \& Moreno; HU, MA.

*Amphinema byssoides (Pers.: Fr.) John Eriksson; CA, GR, HU, MA.

Amylocorticium subsulphureum (P. Karsten) Pouzar; CA.

Amylostereum laevigatum (Fr.) Boidin; CA.

Asterostroma cervicolor (Berk. \& M. A. Curtis)

Massee; GR, J.

Athelia acrospora Jül.; GR.

*Athelia arachnoidea (Berk.) Jül.; GR, J,

Athelia bombacina (Link) Pers.; GR.

Athelia cystidiolophora Parm.; J.

*Athelia decipiens (Höhnel \& Litsch.) John

Eriksson; AL, GR, HU, J.

Athelia epiphylla Pers.; CA, GR, J, MA.

Athelia fibulata M. P. Christ.; AL, GR, J.

Athelopsis glaucina (H. Bourdot \& Galzin) Oberw. ex Parm.; J. MA.

Athelopsis subinconspicua (Litsch.) Jül.; GR.

*Auriculariopsis ampla (Lév.) R. Maire; CO, GR, J, MA.
Botryobasidium candicans John Eriksson; HU.

Botryobasidium laeve (John Eriksson) Parm.; J, MA.

Botryobasidium subcoronatum (Höhnel \& Litsch.) Donk; CA.

Botryohypochnus isabellinus (Fr.) John Eriksson; $\mathrm{HU}, \mathrm{J}, \mathrm{MA}$.

Brevicellicium olivascens (Bres.) Larsson \& Hjortstam; CA, GR.

*Byssomerulius corium (Pers.: Fr.) Parm.; CA, CO, GR, HU, J, MA.

Byssomerulius hirtellus (Burt) Parm.; CA, J, MA.

Ceraceomerulius serpens (Tode: Fr.) Ginns; J.

Ceraceomerulius sublaevis (Bres.) Jül; J.

* Chondrostereum purpureum (Schumacher: Fr.) Pouzar; GR, HU, J, MA.

Clavulicium delectabile (H. Jackson) Hjortstam; CA. Coniophora arida (Fr.) P. Karsten; MA.

Coniophora olivacea (Fr.: Fr.) P. Karsten; GR, J.

*Coniophora opuntiae Tellería; AL.

Coniophora puteana (Schumacher: Fr.) P. Karsten; CA, GR, HU, MA.

Cristinia helvetica (Pers.) Parm.; GR, J.

Crustoderma dryinum (Berk. \& M. A. Curtis) Parm.; J.

Cylindrobasidium evolvens (Fr.: Fr.) Jül.; CO, GR.

Dacryobolus karstenii (Bres.) Oberw.; MA.

*Dacryobolus sudans (Alb. \& Schwein.: Fr.) Fr.; CA, GR, J, MA.

Epithele canariensis (Manjón \& Moreno) Hjortstam, Manjón \& Moreno; CA, GR, MA.

Fibricium rude (P.Karsten) Jülich; CA.

Fibrodontia gossypina Parm.; GR.

Fibulomyces fusoideus Jül.; GR.

Fibulomyces mutabilis (Bres.) Jül.; GR.

Flagelloscypha christinae Agerer; GR.

Galzinia incrustans (Höhnel \& Litsch.) Parm.; CA.

Globulicium hiemale (Laurilia) Hjortstam; CO, HU, MA.

Gloeocystidiellum leucoxanthum (Bres.) Boidin; J.

*Gloeocystidiellum luridum (Bres.) Boidin.; CA, $\mathrm{GR}, \mathrm{J}, \mathrm{MA}$.

Gloeocystidiellum porosum (Berk. \& M. A. Curtis) Donk; J.

Gloiothele lactescens (Berk.) Hjortstam; GR.

* Hymenochaete cinnamomea (Pers.) Bres.; CO, GR, $\mathrm{HU}, \mathrm{J}, \mathrm{MA}$.

Hymenochaete corrugata (Fr.: Fr.) Lév.; HU.

Hymenochaete rubiginosa (Schrader: Fr.) Lév.; CA, GR, J.

Hymenochaete tabacina (Sow.: Fr.) Lév.; HU, MA. Hyphoderma argillaceum (Bres.) Donk; CA.

Hyphoderma cremeo-album (Höhnel \& Litsch.) Jül.; J.

Hyphoderma galacticum Manjón, Moreno \& Hjortstam; MA.

*Hyphoderma malençonii (Manjón \& Moreno) Hjortstam, Manjón \& Moreno; CA, MA.

Hyphoderma multicystidium (Hjortstam \& Ryv.) Hjortstam \& Tellería; AL, GR. 
*Hyphoderma orphanellum (H. Bourdot \& Galzin) Donk; CA, MA.

*Hyphoderma pallidum (Bres.) Donk; CA, HU, MA.

*Hyphoderma praetermissum (P. Karsten) John

Eriksson \& Strid; AL, CA, CO, GR, HU, J.

*Hyphoderma puberum (Fr.) Wallr.; CA, GR, J.

Hyphoderma setigerum (Fr.) Donk; CA, J.

Hyphoderma sibiricum (Parm.) John Eriksson \& Strid; GR, J.

*Hyphodermella corrugata (Fr.) John Eriksson \& Ryv.; CA, GR, J, MA.

*Hyphodontia alutaria (Burt) John Eriksson; CA, MA. Hyphodontia arguta (Fr.) John Eriksson; HU, J.

Hyphodontia aspera (Fr.) John Eriksson; GR.

Hyphodontia cineracea $(\mathrm{H}$. Bourdot \& Galzin) John Eriksson \& Hjortstam; GR.

Hyphodontia juniperi (H.Bourdot \& Galzin) John

Eriksson \& Hjortstam; AL, J.

Hyphodontia pruni (Lasch) Svrcek; CA, MA.

*Hyphodontia quercina (Pers.: Fr.) John Eriksson; CA, HU, J.

*Hyphodontia sambuci (Pers.) John Eriksson; AL, CA, GR, J, MA.

Hyphodontia subalutacea (P. Karsten) John Eriksson; GR.

Hypochnella violacea Anersw. ex Schröeter; J.

* Laeticorticium jonides (Bres.) Donk; CA, GR, HU, J, MA.

* Laeticorticium meridioroseum (Boidin \& Lanquetin) Dueñas \& Tellería; CA, CO, GR, HU, J, MA.

* Laeticorticium pinsapineum Moreno, Manjón \& Hjortstam; MA.

*Laeticorticium polygonioides (P. Karsten) Donk; GR, HU, J, MA.

Laxitextum bicolor (Pers.: Fr.) Lentz.; HU.

Leucogyrophana mollusca (Fr.) Pouzar; J.

*Litschaurella clematidis (H. Bourdot \& Galzin) John Eriksson \& Ryv.; AL, CA, J, MA.

Melzericium udicolum (H. Bourdot) Hauerslev; CA, GR, J.

*Merismodes anomalus (Pers.: Fr.) Singer; GR, J. Mycoacia aurea (Fr.) John Eriksson \& Ryv.; GR, J. Mycoacia uda (Fr.) Donk; GR, HU.

*Peniophora cinerea (Pers.: Fr.) Cooke; CA, GR, J, MA.

*Peniophora incarnata (Pers.: Fr.) P.Karsten; CA, CO, GR, J, MA.

Peniophora junipericola John Eriksson; AL.

Peniophora lilacea H.Bourdot \& Galzin; AL, CO, J.

Peniophora limitata (Chaill.: Fr.) Cooke; J.

*Peniophora lycii (Pers.) Höhnel \& Litsch.; AL, CA, CO, GR, J, MA.

* Peniophora meridionalis Boidin; CA, CO, GR, HU, J, MA.

Peniophora nuda (Fr.) Bres.; AL, J, MA.

Peniophora pini (Schleicher: Fr.) Boidin; MA.
*Peniophora pseudoversicolor Boidin; AL, GR, J, MA.

*Peniophora quercina (Pers.: Fr.) Cooke; AL, CA, $\mathrm{CO}, \mathrm{GR}, \mathrm{HU}, \mathrm{J}, \mathrm{MA}$.

* Peniophora tamaricicola Boidin \& Malençon; AL, CO, GR, MA

Peniophora versicolor (Bres.) Sacc. \& Sydow; GR, J.

Peniophora violaceolivida (Sommerf.) Massee; GR, J.

Phanerochaete aurata (aff.) (H. Bourdot \& Galzin) Burds.; AL.

Phanerochaete avellanea (Bres.) John Eriksson \& Hjortstam; J.

Phanerochaete galactites (H. Bourdot \& Galzin) John Eriksson \& Ryv.; GR, J.

Phanerochaete jose-ferreirae (Reid) Reid; J.

Phanerochaete martelliana (Bres.) John Eriksson \& Ryvarden; CA, HU, J.

*Phanerochaete sanguinea (Fr.) Pouzar; AL, CA, HU, J, MA.

Phanerochaete sordida (P. Karsten) John Eriksson \& Ryv.; CA, GR, J.

* Phanerochaete tuberculata (P. Karsten) Parm.; $\mathrm{CA}, \mathrm{CO}, \mathrm{GR}, \mathrm{J}, \mathrm{MA}$.

Phlebia albida Post in Fr.; GR, J.

Phlebia bresadolae Parm.; GR, HU, J.

Phlebia cornea (H. Bourdot \& Galzin) Parm.; J.

Phlebia cremeo-alutacea (Parm.) Larsson \& Hjortstam.; J.

* Phlebia georgica Parm.; MA.

Phlebia lilascens (H. Bourdot \& Galzin) John Eriksson \& Hjortstam; MA.

Phlebia livida (Pers.: Fr.) Bres.; CA, J.

Phlebia nitidula (P. Karsten) Ryv.; GR, J.

Phlebia radiata $\mathrm{Fr}$.; HU.

Phlebia ryvardenii Hallenb. \& Hjortstam.; J.

Phlebia subulata John Eriksson \& Hjortstam.; J.

Phlebia tremellosa (Schrader: Fr.) Nakasone \& Burdsall.; CA, GR, HU, J.

Phlebiella pseudotsugae (Burt) Larsson \& Hjortstam; MA.

*Phlebiella vaga (Fr.) P. Karsten; GR, HU, J, MA.

*Phlebiopsis gigantea (Fr.: Fr.) Jül.; GR, HU, J.

Phlebiopsis ravenelii (Cooke) Hjortstam; CA, J.

Piloderma byssinum (P. Karsten) Jül.; J, MA.

Podoscypha multizonata (Berk. \& Br.) Pat.; HU, CA.

Pteridomyces subsphaericosporus Boidin, Duhem \& Gilles; J.

*Pulcherricium caeruleum (Schrader: Fr.) Parm.; $\mathrm{CA}, \mathrm{CO}, \mathrm{GR}, \mathrm{HU}, \mathrm{J}, \mathrm{MA}$.

*Radulomyces confluens (Fr.: Fr.) M. P. Christ.;

*Radulomyces molaris (Chaill.: Fr.) M. P. Christ.; CA, GR, HU, J, MA.

*Schizophyllum commune Fr.; AL, CO, GR, HU, J. Scopuloides hydnoides (Cooke \& Massee) Hjortstam \& Ryv.; GR.

Scytinostroma aluta Lanquetin; J, MA.

Scytinostroma mediterraneense Boidin \& Laquetin; 
GR, MA.

Serpula himantioides (Fr.: Fr.) P. Karsten; CA.

Sistotrema brinkmannii (Bres.) John Eriksson; AL, $\mathrm{CO}, \mathrm{GR}, \mathrm{J}$.

Sistotrema coroniferum (Höhnel \& Litsch.) Donk; GR.

Sistotrema diademiferum (H. Bourdot \& Galzin) Donk; GR, J.

Sistotrema octosporum (Schröeter ex Höhnel \& Litsch.) Hallenberg; HU, MA.

Sistotrema sernanderi (Litsch.) Donk; CA.

Sistotremastrum suecicum Litsch. ex John Eriksson; MA.

Steccherinum ciliolatum (Berk. \& M. A. Curtis) Gilbertson \& Buding; AL, J.

Steccherinum fimbriatum (Pers.: Fr.) John Eriksson; HU, J, MA.

*Steccherinum ochraceum (Pers.: Fr.) S. F. Gray; CA, CO, GR, HU, J, MA.

Steccherinum queletii (H. Bourdot \& Galzin) Hallenberg \& Hjortstam; AL, MA.

* Stereum gausapatum (Fr.) Fr.; CA, CO, GR, HU, J.

*Stereum hirsutum (Willd.: Fr.) S. F. Gray; AL, CA, CO, GR, HU, J, ,MA.

Stereum ochraceoflavum (Schwein.) Ell.; CA.

Stereum ostrea (Nees: Fr.) Fr.; GR.

Stereum reflexulum Reid; J.

Stereum rugosum (Pers.: Fr.) Fr.; J.

Stereum sanguinolentum (Alb. \& Schwein.: Fr.) Fr.; GR, MA.

Subulicystidium longisporum (Pat.) Parm.; CA, J.

Tomentella atramentaria Rostrup; GR.

Tomentella bresadolae (Brinkm.) H. Bourdot \& Galzin; GR.

Tomentella brevispina (H. Bourdot \& Galzin) M. J. Larsen; GR.

Tomentella bryophila (Pers.) M. J. Larsen; GR, J, MA.

Tomentella lateritia Pat.; J.

Tomentella nitellina H.Bourdot \& Galzin; CO.

Tomentella ruttneri Litsch.; GR.

Tomentella sublilacina (Ell. \& Holway) Wakef.; GR.

Tomentella violaceofusca (Sacc.) M. J. Larsen; J.

Tomentella umbrinospora M. J. Larsen; MA.

Tomentellastrum alutaceoumbrinum (Bres.) M. J. Larsen; GR.

Tomentellastrum badium (Link) M. J. Larsen; GR.

Tomentellopsis submollis (Svreck) Hjortstam; CA.

Trechispora alnicola (H.Bourdot \& Galzin) Liberta; J.

Trechispora confinis (H.Bourdot \& Galzin) Liberta; $\mathrm{HU}$.

Trechispora farinacea (Pers.: Fr.) Liberta; CA, GR.

Trechispora microspora (P. Karsten) Liberta; J.

Tubulicium vermiferum (H. Bourdot) Oberw.; MA.

Tubulicrinis calothrix (Pat.): Donk; CA.

Tubulicrinis gracillimus (Ell. \& Ev. ex D. P. Rogers
\& H. Jackson) C. Cunn.; CA, GR.

Tubulicrinis sororius (H. Bourdot \& Galzin) Oberw.; JA, MA.

Tubulicrinis subulatus (H. Bourdot \& Galzin) Donk.; CA.

Tubulicrinis thermometrus (G.Cunn.) M. P. Christ.; MA.

Uncobasidium calongei (Tellería) Hjortstam \& Tellería.; AL.

Uthatobasidium fusisporum (Schröeter) Donk; AL. Vararia ochroleuca (H. Bourdot \& Galzin) Donk; AL.

Vesiculomyces citrinus (Pers.) Hagströn; CA.

*Vuilleminia comedens (Nees: Fr.) R. Maire; CA, CO, GR, J.

Vuilleminia corylii (aff.) Boidin; HU.

Vuilleminia cystidiata Parm.; GR, J.

*Vuilleminia macrospora (Bres.) Hjortstam; AL, GR, HU, J.

*Vuilleminia megalospora Bres.; CO, GR, J, MA. Xenasma pruinosum (Pat.) Donk; CO.

Xenasma pulverulentum (Litsch.) Donk; J.

Xylobolus subpileatus (Berk. \& M. A. Curtis) Boidin; CA.

\section{APHYLLOPHORALES PORADOS:}

*Abortiporus biennis Murrill; CO, GR, HU, J, MA. Anomoporia myceliosa (Peck) Pouzar.; HU.

Antrodia albidoides David \& Dequatre; CA, HU, J. Antrodia albobrunnea (Romell) Ryv.; J.

Antrodia sinuosa (Fr.) Ryv.; MA

Antrodia xantha (Fr.) Ryv.; GR.

*Antrodiella romelli (Donk) Niemelä; GR, HU.

*Bjerkandera adusta (Willd.: Fr.) P. Karsten; AL, CA, CO, GR, HU, J, MA.

Bjerkandera fumosa (Pers.: Fr.) P. Karsten; CA.

Byssoporia terrestris (Pers.: Fr.) Larsen \& Zak; GR.

Cerioporia purpurea (Fr.) Donk; AL, GR.

Cerioporia reticulata (Fr.) Domanski; GR.

Cerioporiopsis mucida (Pers.: Fr.) Gilbn. \& Ryv.; CA, MA.

Cerioporiopsis resinascens (Romell) Domanski; J.

*Cerrena unicolor (Bull.: Fr.) Murr.; GR, HU, J, MA.

*Coriolopsis gallica (Fr.) Ryv.; AL, CO, GR, HU, MA.

Daedalea quercina $\mathrm{Fr}$.; $\mathrm{CO}, \mathrm{GR}, \mathrm{J}$.

*Dichomitus campestris (Quél.) Domanski \& Orliez; CA, HU, J.

Dichomitus squalens (P. Karsten) Reid; AL, HU.

*Fistulina hepatica Schaeff.; CO, GR, HU, J.

Fomes fomentarius (L.: Fr.) Kickx; CO, GR, HU, J.

*Fomitopsis pinicola (Swartz: Fr.) P. Karsten; AL, CA, GR, HU, J, MA.

*Ganoderma adspersum (Schulz.) Donk; AL, GR, 
HU, MA.

Ganoderma chalceum (Cooke) Steyaert; GR.

Ganoderma lipsiense (Batsch) Atkinson; CO.

*Ganoderma lucidum (W. Curt.: Fr.) P. Karsten; $\mathrm{AL}, \mathrm{CO}, \mathrm{GR}, \mathrm{HU}, \mathrm{J}$.

*Ganoderma resinaceum Boud.; CA, CO, GR, HU, MA.

Gelatoporia pannocincta (Romell) Niemelä; HU.

Gloeophyllum abietinum (Bull.: Fr.) P. Karsten; J.

*Gloeophyllum sepiarium (Wulf.: Fr.) P. Karsten; $\mathrm{CO}, \mathrm{GR}, \mathrm{J}$.

*Gloeophyllum trabeum (Pers.: Fr.) Murr.; AL, GR, J. Gloeoporus dichrous (Fr.) Bres.; CA, GR, HU.

Grifola frondosa (Dicks.: Fr.) S. F. Gray; GR, HU, MA.

Hapalopilus rutilans (Pers.: Fr.) P. Karsten; HU.

Heterobasidion annosum Bres; CA, MA.

Hexagonia nitida Dur. \& Mont.; CO, HU.

Inonotus dryadeus (Pers.: Fr.) Murr.; CO.

*Inonotus hispidus (Bull.: Fr.) P. Karsten; AL, CO, GR, HU, J.

Inonotus radiatus (Sow.: Fr.) P. Karsten; CA.

*Inonotus tamaricis (Pat.) R. Maire; AL, CO, GR, HU, J.

Laetiporus sulphureus (Bull.: Fr.) Murr.; CO, HU, J.

Lenzites betulina (L.: Fr) Fr.; GR, HU.

Oligoporus caesius (Schrad.: Fr.) Gilbn. \& Ryv.; MA.

Oligoporus fragilis (Fr.) Gilbn.; MA.

Oligoporus hibernicus (Berk. \& Br.) Gilbn. \& Ryv.; CA.

Oligoporus leucomallellus (Murr.) Gilbn. \& Ryv.; MA.

Oligoporus subcaesius (David) Gilbn. \& Ryv.; J.

Oxyporus corticola (Fr.) Ryv.; GR.

Oxyporus populinus (Schum.: Fr.) Donk.; CA

Perenniporia medulla-panis (Jacq.: Fr.) Donk.; HU.

Perenniporia rosmarini David \& Malençon.; AL, $\mathrm{CO}, \mathrm{J}$.

Phaeolus schweinitzii (Fr.) Pat.; GR, HU, J.

Phellinus conchatus (Pers.: Fr.) Quél.; GR.

Phellinus hartigii (Allesch. \& Schnabl.) Bond.; MA.

Phellinus igniarius (L.: Fr.) Quél.; AL, J.

*Phellinus pini (Thore.: Fr.) A. Ames; AL, GR, J.

Phellinus punctatus (Fr.) Pilát; GR.

Phellinus ribis (Fr.) Quél.; GR.

Phellinus rimosus (Berk.) Pilát.; GR, HU.

*Phellinus robustus (P.Karsten) H. Bourdot \& Galzin; CO, GR, J.

*Phellinus torulosus (Pers.) H. Bourdot \& Galzin; AL, CA, CO, GR, HU, J.

Phellinus tuberculosus (Baumg.) Niemelä; AL, GR, HU, J.

*Polyporus arcularius Batsch: Fr.; AL, CA, CO, GR, HU, J.

Polyporus brumalis (Pers.: Fr.) Fr.; CA.

Polyporus melanopus (Schwein.) Fr.; GR.
*Polyporus meridionalis (David) Jahn; CO, GR, HU, J, MA.

*Polyporus squamosus Huds. Fr.; CO, GR, HU, J. Polyporus tuberaster (Jacq.) Fr.; CA, HU.

Pycnoporus cinnabarinus (Jacq.: Fr.) P. Karsten; HU.

*Schizopora paradoxa (Fr.) Donk; CA, GR, HU, J, MA.

Skeletocutis nivea (Jungh.) Keller; J.

Skeletocutis percandida (Malençon \& Bertault) Keller; HU, J.

Skeletocutis subincarnata (Peck) Keller; J.

*Skeletocutis subsphaerospora David; CA, MA.

Spongipellis pachyodon (Pers.) Kotl. \& Pouzar; $\mathrm{HU}$.

Trametes pubescens (Schum.: Fr.) Pilát; CA, GR, J.

*Trametes trogii Berk.; AL, CA, GR, HU, J, SE.

*Trametes versicolor (L.: Fr.) Pilát; CA, CO, GR, HU, J, MA, SE.

Trechispora mollusca (Pers.: Fr.) Liberta; HU.

Trichaptum abietinum (Dicks.: Fr.) Ryv.; GR, MA.

*Trichaptum biforme (Fr. in Kl.) Ryv.; CA, GR, HU, J.

*Trichaptum fusco-violaceum (Ehrenb.: Fr.) Ryv.; GR, HU, J, MA.

\section{BIBLIOGRAFÍA}

ARISTA, M., F. J. HERRERA y S. TALAVERA 1997- Biología del Pinsapo. Consejería de Medio Ambiente. Junta de Andalucía.

CALONGE, F. D. y J. A. ORIA DE RUEDA -1988Aportación a la micoflora de la provincia de Almería. Bol. Soc. Micol. Madrid 12: 93-106.

CALONGE, F. D. y L. ROMERO DE LA OSA 1997- Podoscypha multizonata (Berk. \& Br.) Pat., un hongo casi desconocido en España. Bol. Soc. Micol. Madrid 22: 295-296.

CALONGE, F. D. y M. T. TELLERÍA -1980Introducción al conocimiento de los hongos de Doñana (Huelva, España). Lazaroa 2: 271-326.

GÓMEZ, J., A. ORTEGA, y B. MORENO-ARROYO -1995- Contribución al catálogo micológico de la provincia de Córdoba. I. Catálogo del Parque Natural de las Sierra Subbéticas y su entorno (Córdoba, España). Bol. Soc. Micol. Madrid 20: 225-267.

GOSLEE, S. C., R. P. BROOKS, \& C. A. COLE 1997- Plants as indicators of wetland water source. Plant Ecology 131: 199-206.

HJORTSTAM, K., J. L. MANJÓN, \& G. MORENO -1988- Notes on select Corticiaceous Fungi from 
Spain and north Africa. Mycotaxon 33: $257-$ 263.

HJORTSTAM, K., M. T. TElleríA, L. RYVARDEN, \& F. D. CALONGE - 1981- Notes on the Aphyllophorales of Spain II. Nova Hedwigia 34(3-4): 525-538.

HONRUBIA, M. y X. LLIMONA -1982- Aportación al conocimiento de los hongos del S.E. de España. VIII. Afiloforales (Basidiomicetes). Collect. Bot. 13(2): 491-532.

JIMENEZ-ANTONIO, F. -1994- Contribución al estudio de los hongos de la provincia de Jaén. I. Bol. Soc. Micol. Madrid 19: 111-154.

MALENÇON, G. -1968- Contribution a la flore mycologique de L'andalousie. Collect. Bot. VII(2) 40: 707-725.

MALENÇON, G. \& R. BERTAULT -1976Champignons de la Péninsule Ibérique. V. Catalogne, Aragon, Andalousie. Acta Phytotaxonomique Barcinonensia 19: 5-67.

MALENÇON, G \& X. LLIMONA -1980Champignons de la Péninsule Ibérique. VI. Est et Sud-Est. Anal. Universidad Murcia, Ciencias, XXXIV(1-2-3-4): 47-135.

MANJÓN, J .L. y G. MORENO -1983- Estudios sobre Aphyllophorales. III. Fructificaciones sobre Abies Pinsapo Boiss. Cryptogamie, Mycol. 4: $145-156$.

MORENO, G. y J. L. MANJÓN -1987- Nuevas aportaciones al estudio de los Aphyllophorales de la vegetación relicta del Abies Pinsapo. Bol. Soc. Micol. Madrid 11(2): 261-266.

ORTEGA, A., F. ESTEVE-RAVENTÓS, E. HORAK y G. MORENO - 1996- Aportación al catálogo de los macromicetos del área potencial del Abies Pinsapo en España. Bol. Soc. Micol. Madrid 21: 219-249.

ORTEGA, A., R. GALÁN y M. T. TELLERÍA 1982- Aportación al estudio de los hongos de Andalucía VII. Catálogo de los Aphyllophorales de la provincia de Granada. Bull. Soc. Micol. Castellana 7: 53-68.

ORTEGA, A., G. MORENO y ESTEVERAVENTÓS, F. -1997-Contribución al estudio micológico del Parque Natural de los Alcornocales (Andalucía, España). Bol. Soc. Micol. Madrid 22: 219-272.

ORTEGA, A., VIZOSO, M. T. y CONTU, M. 1991- Notas sobre la micoflora xero-termófila y sabulícola de Andalucía. (primera parte). Doc.
Mycol. 21 (82): 19-42.

RIVAS-MARTÍNEZ, S. - 1996- Clasificación bioclimática de la Tierra. Folia Botánica Matritensis 16: 1-32.

ROMERO DE LA OSA, L. -1992- Contribución al estudio de los hongos de la sierra de Aracena (Huelva). II. Bol. Soc. Micol. Madrid 16: 205213.

ROMERO DE LA OSA, L.-1993- Contribución al estudio de los hongos de la sierra de Aracena (Huelva). III. Bol. Soc. Micol. Madrid 18:135144.

ROMERO DE LA OSA, L.-1996- Contribución al estudio de los hongos de la sierra de Aracena (Huelva). IV Bol. Soc. Micol. Madrid 21:7-30.

TELLERÍA, M. T. -1984- De Aphyllophoralibus in Hispania provenientibus ordinati comentarii, II. Anal. Jard. Bot. Madrid 41(1): 25-33.

TELLERÍA, M. T. -1985- De Aphyllophoralibus in Hispania provenientibus ordinati comentarii, III. Anal. Jard. Bot. Madrid 42(1): 49-60.

TELLERÍA, M. T., -1987-Aphyllophorales (Basidiomycotina) of the Iberian and north African fir woodlands (Abies Pinsapo). A contribution to their study. Nova Hedwigia 44(12): 105-120.

TELLERÍA, M. T. -1990- Annotated list of the Corticiaceae sensu lato (Aphyllophorales, Basidiomycotina), for Peninsular Spain and Balearic Islands. J.Cramer. Stuttgart.

TELLERÍA, M. T. - 1991- Additions and corrections of the annotated list of the Iberian Corticiaceae (Aphyllophorales, Basidiomycotina). I. Nova Hedwigia 53(1-2): 229-253.

VIZOSO, M. T. \& ORTEGA, A. -1992- Notes on palm-growing Macromycetes. Doc. Mycol. 22 (86): 31-39.

VIZOSO, M. T., ORTEGA, A. Y MANJÓN, J. L. 1991- Primera contribución al conocimiento de los Aphyllophorales S.L. de las comunidades naturales de Andalucía. Bol. Soc. Micol. Madrid 15: 153-164.

Aceptado para su publicación en abril de 2000

Dirección de los autores. Dpto. Biología Vegetal, Facultad de Ciencias, Campus de Fuentenueva. Universidad de Granada. 18001 GRANADA. Tf/ Fax : 958-243234/ 958-243254; E-mail: genfito@ goliat.ugr.es; aortegad@ugr.es 